\title{
CLINICAL OBSERVATIONS ON THE EVENTS PRECEDING THE APPEARANCE OF RHEUMATIC FEVER ${ }^{1,2}$
}

\author{
By EDWARD F. BLAND AND T. DUCKETT JONES
}

(From the House of the Good Samaritan, Boston)

(Received for publication May 27, 1935)

The frequent association between tonsillitis and upper respiratory tract infection and subsequent rheumatic fever has been observed and commented upon by many students of the disease. It is generally agreed that this relationship between the preceding infection and subsequent rheumatic fever is too consistent to be merely a chance occurrence. This important phase of the natural course of rheumatic fever has been commented upon by Coburn $(1,2)$, Schlesinger (3), Collis (4), Sheldon (5), Gibson, Thomson, and Stewart (6), Boas and Schwartz (7), Hiller and Graef (8), and others. In general, we agree with these investigators both as regards the association between infection of the upper respiratory tract and rheumatic fever, as well as to apparent epidemics of rheumatic fever, following prevalent respiratory infection in groups of closely associated individuals. The percentage of instances of rheumatic fever preceded by recent upper respiratory infection may be as high as 75 per cent. Not only does this relationship hold for the initial appearance of the disease, but also for subsequent recurrences or recrudescenses. It is of further interest that in most instances there exists, as pointed out by Schlesinger (3), a well defined latent period varying from a few days up to four weeks between the febrile respiratory tract infection and the occurrence. of the clinical signs and symptoms of rheumatic fever. Less frequently the disease appears to be ushered in or occurs concurrently with the respiratory infection.

Because of the obscure nature of the etiological factors involved, attention has been directed, and we believe rightly, to this so-called respiratory phase of the disease and the subsequent latent period. Its analogy to the sequence of events occurring in serum sickness and other frankly al-

\footnotetext{
1 Presented at the meeting of the American Society for Clinical Investigation, Atlantic City, April 30, 1934.

2 The expenses of this study have been defrayed by a grant from The Commonwealth Fund.
}

lergic conditions has been commented upon by Zinsser (9), Swift (10), and others. Exhaustive study has been centered upon the cultural characteristics of the respiratory flora during these preceding infections in an attempt to establish a specific causative agent. In so far as we are aware no proof has yet been presented that any organism found in the upper respiratory tract is the cause of rheumatic fever.

It is the purpose of this paper to present certain clinical observations on rheumatic fever which are of interest and may be of importance in future considerations of possible factors in the causation of the disease. During the past thirteen years at the House of the Good Samaritan there has been an opportunity to observe the clinical course of a large number of children and adolescents (over 1,200) during various phases of rheumatic fever in a hospital where they receive bed care until all clinical and laboratory evidence of infection has subsided. The average period of observation in the hospital is four months, but not infrequently is as long as several years. During this period of prolonged observation, the evidence of persistent low-grade and sub-clinical infection has been largely that of a slight fever (rectal), subcutaneous nodules, erythema multiforme, a moderate leukocytosis, increased sedimentation rate of the red blood cells, or prolongation of the auriculoventricular conduction time. It is in this mildly active group that we have observed repeatedly the importance of respiratory infection in precipitating relapses or recrudescences of the clinical manifestations of the acute disease. Figure 1 shows the bedside chart of a boy, aged thirteen, convalescing from recent rheumatic fever in whom the clinical and laboratory evidence of active disease had subsided. Following the febrile respiratory infection shown on the chart and after a latent period of two weeks, the boy developed fulminant rheumatic fever with pericarditis and congestive failure. $\mathrm{He}$ died at the end of one 


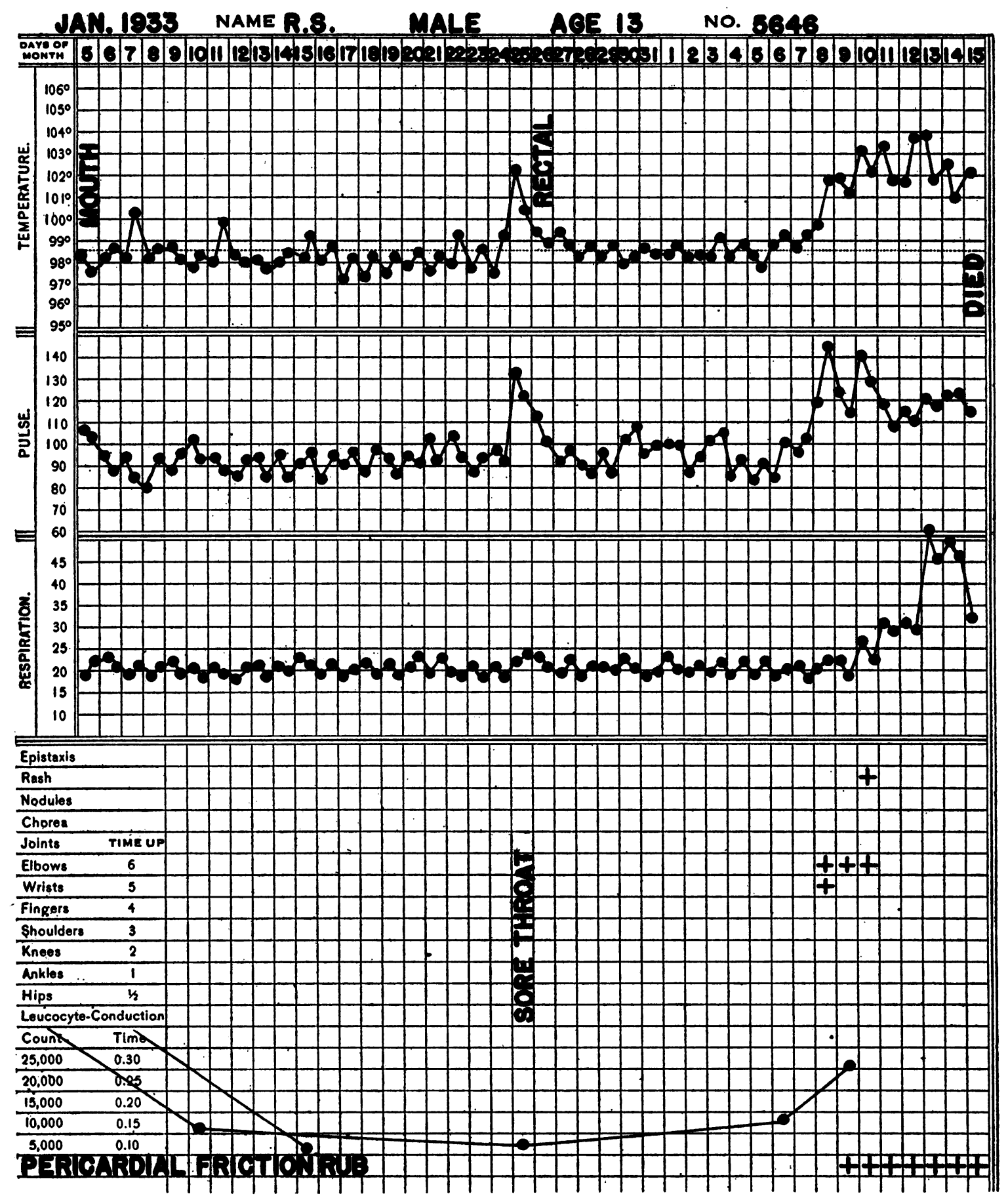

Fig. 1. Bedside Chart Showing the Relationship Between Upper Respiratory Infection and a Subsequent Recrudescence of Fulminant Rheumatic Fever. 
week, and rheumatic fever was confirmed by autopsy.

Recurrent rheumatic fever has been observed at times following infections other than tonsillitis or pharyngitis. Scarlet fever apparently is frequent as a precipitating factor and further data on this interesting relationship will be published subsequently. These episodes are closely related to streptococcus infection. Instances of other precipitating infections, frequently associated with or subsequent to ordinary respiratory infection noted in our series are: "pneumonia" 4 , otitis media 5 , measles 3 , and erysipelas 1 . One might add here single episodes, seemingly independent of respiratory infection, which we have observed to precede recurrent rheumatic fever; namely, pyelitis, herpes zoster, and a febrile reaction to the Schick test.

We have been impressed by the occurrence, now and then, of an equally striking though less frequent association between other (non-infectious) and apparently non-specific episodes and subsequent recurrent rheumatic fever. These episodes exhibit a suggestively similar relationship to the occurrence of subsequent rheumatic fever as has previously been noted between respiratory infection and the acute phase of the disease. Subsequent observations herewith recorded appear to throw additional light upon this interesting but imperfectly understood phenomenon.

\section{Transient and unexplained temperature}

When recrudescences followed respiratory infection, a mild fever of two or three degrees at the time of the infection has been the rule. In contrast, however, we have seen on several occasions a transient fever of two or three degrees for a few hours, unassociated with evident infection or other manifest cause and apparently precipitating after the usual "latent period" a recrudesence of typical rheumatic fever. Figure 2 shows the course of the disease in a child, four years of age, who appeared to be convalescing satisfactorily from rheumatic fever which began twelve months previously. On February 26, as indicated in the chart, there occurred a transient and entirely unexplained elevation of the temperature and pulse for a few hours, followed in two weeks by fulminant rheumatic fever associated with pericar- ditis, congestive failure and death eleven days after the onset. The response of the temperature to salicylates without clinical improvement in the patient is of interest. Postmortem examination confirmed the clinical impression of severe and fulminant rheumatic fever. In the light of previous observations on the apparent relationship of respiratory infection to acute rheumatic fever the association here seems equally striking, but in this instance recrudescence followed a temperature reaction alone. The relationship appears even more convincing when we consider that the bedside chart for the preceding six months had shown a consistently normal temperature and steady pulse rate.

\section{Accidents and operations}

In our experience this same sequence of events has been precipitated apparently by accidents involving fractured bones or sprained joints. Surgical operations have also appeared to reactivate, occasionally, a latent rheumatic infection. It is not an infrequent experience in general hospitals to observe the recurrence of the signs and symptoms of rheumatic fever following tonsillectomy. Indeed, on rare occasions, the initial attack of rheumatic fever is observed following this procedure. We have rarely encountered such a series of events (in 7 instances), since it has been our policy to delay tonsillectomy until all clinical and laboratory evidence of active rheumatic fever has disappeared. This precaution is probably responsible for the small number of recurrences that we have observed after tonsillectomy. The explanations for the reappearance of rheumatic fever following tonsillectomy have been many and inconclusive. Appendectomy has seemingly been related in 10 instances to a subsequent attack of rheumatic fever. Frequently the appendix, at the time of removal, has appeared to be normal. It may be that some of these cases, at least, exemplify an onset of rheumatic fever with abdominal symptoms rather than a true initiation or reactivation of the rheumatic process by the surgical procedure. Of this we have no proof.

The clinical course of the disease as illustrated in Figure 3 is of unusual interest in this connection. A girl of thirteen years had had four pre- 


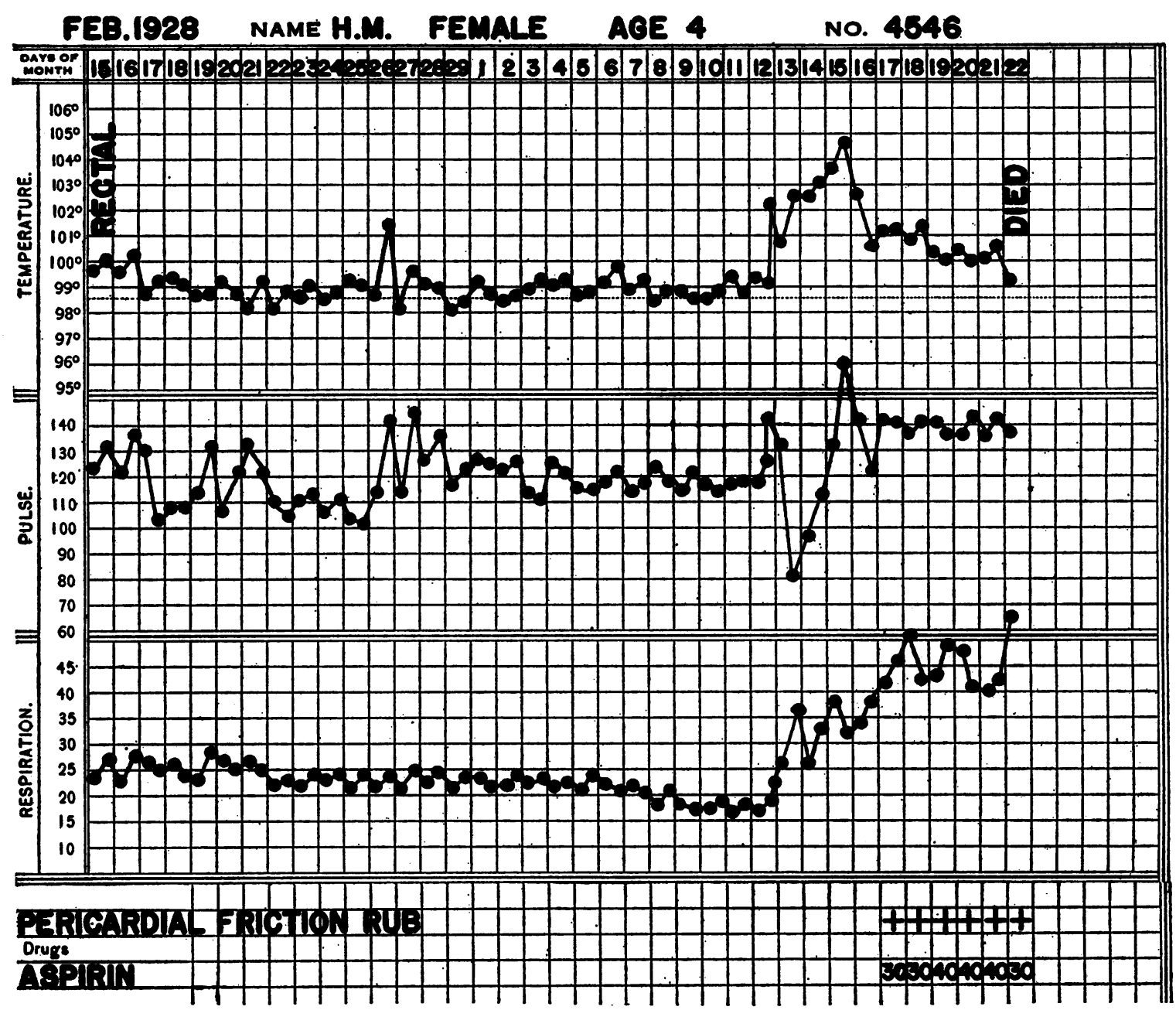

Fig. 2. A fatal Recrudescence of Rheumatic Fever Two Weeks After a Transient and Unexplained FEVER.

vious attacks of rheumatic fever, the last having subsided at the age of twelve, one year before the recorded events transpired. In the interim she had been well. She entered the Orthopedic Service of the Massachusetts General Hospital for surgical correction of an old ankle injury. On March 28 (Figure 3), under ethylene anesthesia, an arthrodesis of the ankle was performed. Immediately following the operation a persistent and increasing fever appeared for which there was no obvious explanation. On the ninth day, because of the continued and unexplained fever, the wound was explored under gas and oxygen anesthesia and found to be entirely clean. Cultures were sterile. The second operation appeared to aggravate the temperature and leukocytosis, the true nature of which was not suspected until a to and fro pericardial friction rub was discovered together with signs of early congestive heart failure. Subsequently, the electrocardiogram showed a delayed auriculoventricular conduction time. The patient's condition, after a rather stormy course, slowly improved on salicylates and digitalis, and she was discharged from the hospital a month later clinically well but still with a leukocytosis of 13,800. There were no joint pains at any time. The evidence seems clear that, in this instance, the general disturbance resulting from the anesthesia and surgical operation in a rheumatic child, who was clinically well, was responsible for the reactivation of a latent rheumatic process. We believe this to be more than a coincidence, in 


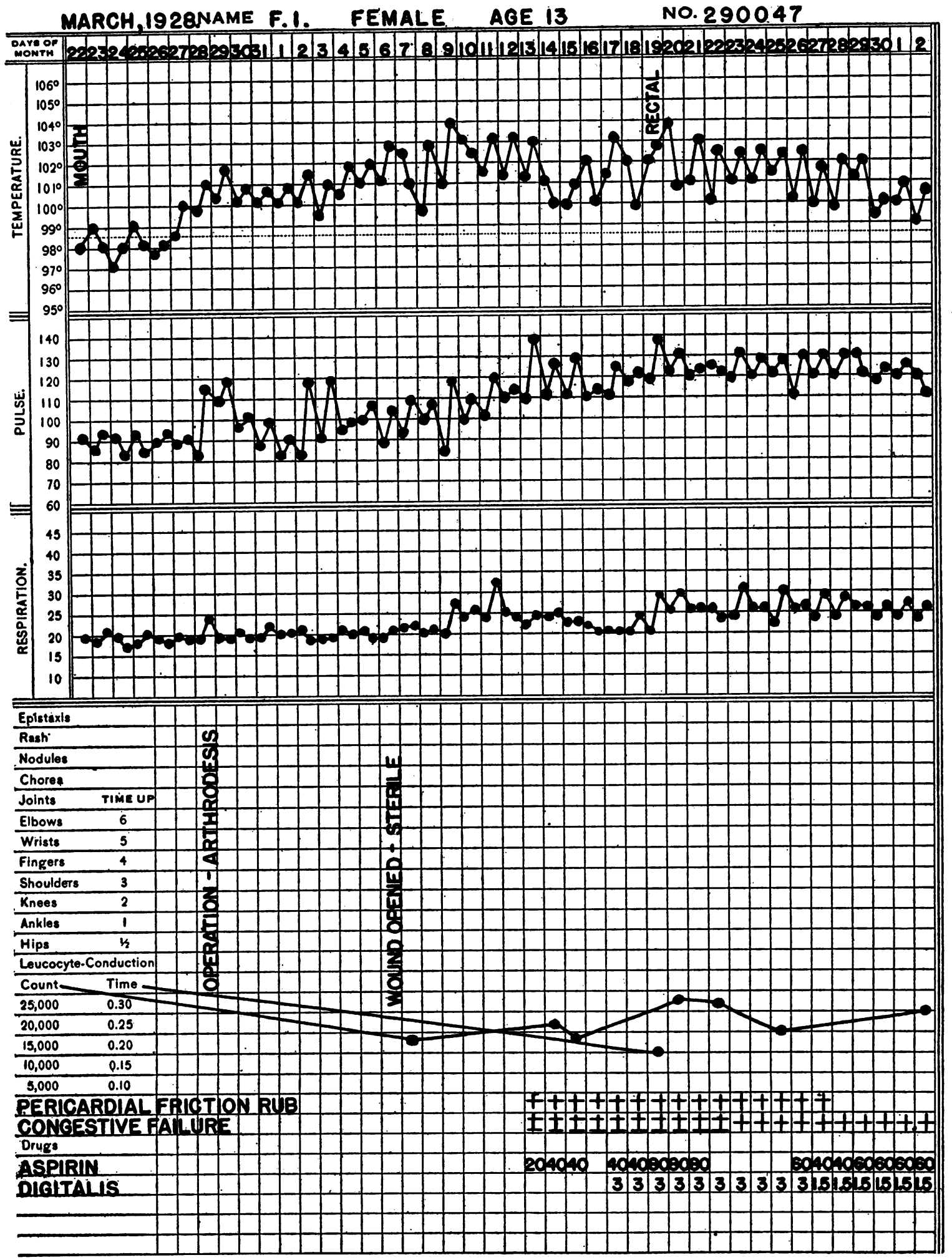

Fig. 3. A Recurrence of Rheumatic Fever Apparently Precipitated by a Surgical Operation under General Anesthesia. 
view of the entire absence of infection either in the respiratory tract or elsewhere.

There remains to be recorded a crucial observation on a girl aged twenty years who entered the medical wards (E. M. 319,881) of the Massachusetts General Hospital with severe chorea and minimal rheumatic heart disease. For the preceding six months she had been in relatively poor health, with vague and repeated joint pains and occasional nosebleeds, prior to the onset of chorea two months before entry. Because of the increasing severity of the chorea, in spite of rest in bed and sedatives, she was given protein shock therapy. After the third daily injection of 0.1 cc. of stock typhoid-paratyphoid vaccine intravenously, she exhibited an unusual and alarming rise in temperature to $108.9^{\circ} \mathrm{F}$. (rectal), which subsided promptly after ice packs had been applied. No further immediate complications appeared, and the severity of the chorea was considerably reduced. Two weeks after the last protein shock the patient developed a low-grade fever of from two to three degrees, complained of multiple joint pains, and an aortic diastolic murmur, not previously noted, was heard. The electrocardiogram, taken for the first time, showed a delay in auriculoventricular conduction with a $\mathrm{P}-\mathrm{R}$ interval of 0.30 second. The joint pains and fever responded promptly to salicylates, and the patient left the hospital against advice. Twelve months later she reported at our request for a follow-up examination. She was in good health and had had no further recurrences of rheumatic fever. There was evidence on physical examination of slight cardiac enlargement with minimal disease of mitral and aortic valves. It occurred to us, in retrospect, that perhaps in this instance by reactivation a low-grade rheumatic fever had been brought to a clinical level as a result of the therapeutic injections of typhoid-paratyphoid vaccine.

\section{Reactions after typhoid-paratyphoid vaccine}

Numerous observers have reported favorably upon the use of intravenous typhoid-paratyphoid vaccine (as an agent for non-specific protein shock) in the treatment of a large number and variety of diseases. This procedure is widely used, especially in treating diseases of unknown etiology. It seemed logical and justifiable to use this (typhoid-paratyphoid) vaccine intravenously in patients with rheumatic fever in a dosage comparable to that of usual therapeutic procedures. The value of this therapeutic measure has not been completely studied in rheumatic fever. In addition, it was thought possible that one might secure information concerning the rôle of nonspecific fever in patients with rheumatic fever, as well as the significance of the febrile episodes often observed before recurrences. The procedure determined upon was to give a single intravenous dose of stock typhoid-paratyphoid vaccine (0.1 cc. of vaccine containing $250,000,000$ organisms). This quantity usually results in a moderate temperature reaction and a chill. The method of repeated doses of typhoid-paratyphoid vaccine, as used by Sutton and her co-workers in the treatment of chorea (11) and as has been employed in rheumatic fever (12), has not been followed.

The patients chosen for this procedure were carefully selected. They were chiefly adolescent individuals who had shown no tendency to progressive cardiac disease in spite of frequent attacks of rheumatic fever. Most of the cases had had a number of attacks during a long period of observation. In addition, all were in excellent general physical condition but exhibited evidence, chiefly from laboratory examination, of low-grade chronic rheumatic fever. Save for recent joint pain, occasional epistaxis and an occasional transient erythema marginata in three cases, the evidence of continued rheumatic fever was based entirely on abnormal laboratory findings. A few, as will be pointed out later, had shown in the past a clear relationship between respiratory infection and a subsequent attack of rheumatic fever. We desire to record in some detail the results of these few observations.

\section{Case I}

R. M., H.G.S. 5614, female, age 16, gave a history of frequent attacks of rheumatic fever since the age of six years, usually following a cold but on two occasions after pneumonia. On entrance to the hospital, in November, 1932, she had laboratory evidence of low-grade rheumatic infection with moderate enlargement of the heart and the murmurs of mitral stenosis and regurgitation and slight aortic regurgitation.

Observation I. In May, 1933, Figure 4, she developed 
MaY, 1933 name R.Meg. female AGE 16 No. 5614

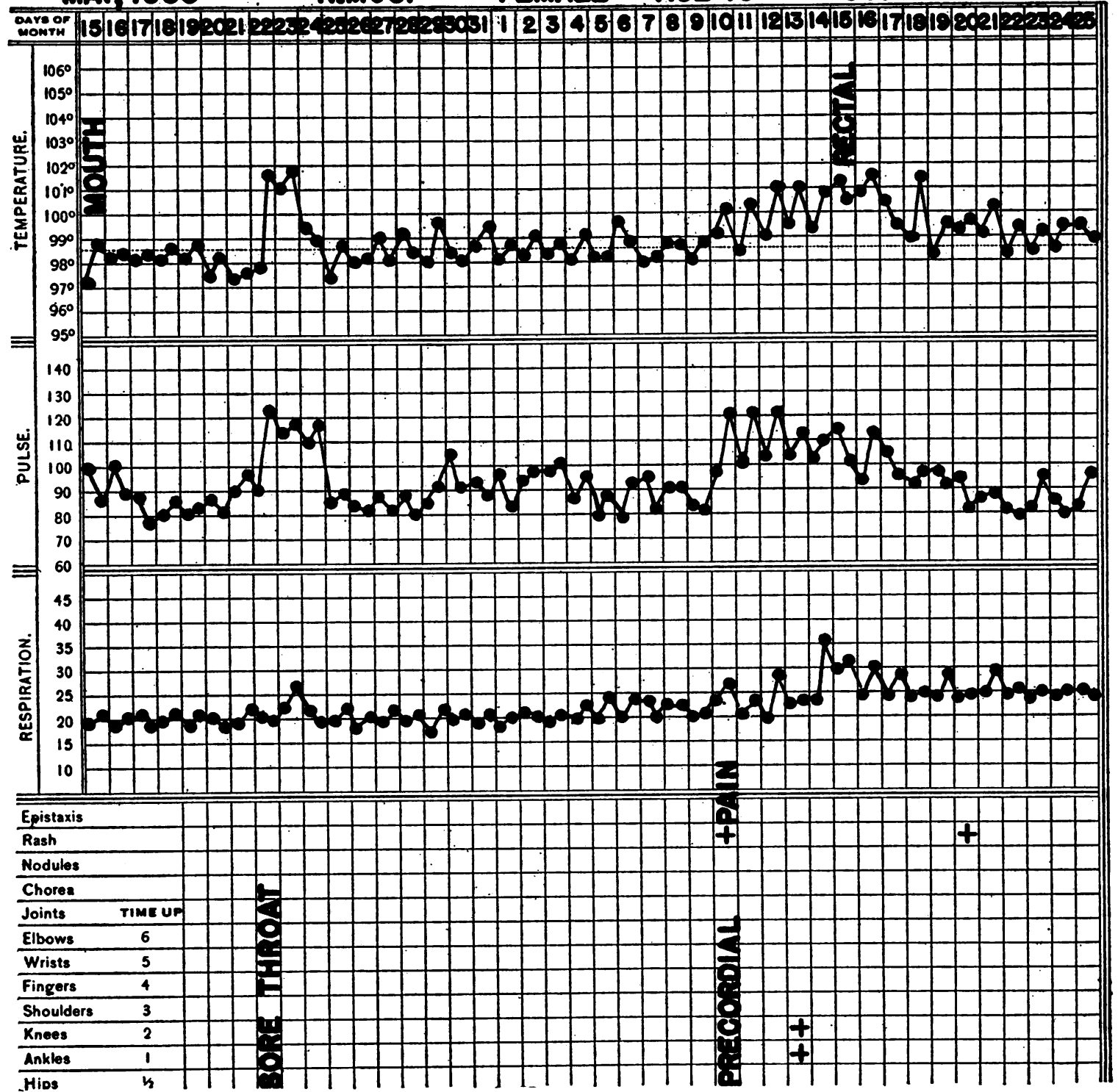

Fig. 4. A Recurrence of Rheumatic Fever Three Weers After Respiratory Infection.

a sore throat and fever for two days. After a latent period of three weeks a recrudescence of clinically active rheumatic fever appeared, associated with fever, joint and precordial pain and erythema multiforme. Hemolytic streptococci were present in throat cultures throughout both the respiratory and the rheumatic fever phases of this observation.

Observation II. After this episode had subsided she was given, in July, 1933, an intravenous injection of 0.1 cc. of stock typhoid-paratyphoid vaccine, which resulted in a mild and transient temperature reaction as indicated in Figure 5. After a latent period of three weeks a typical recrudescence of clinically active rheumatic fever appeared with fever, multiple polyarthritis and erythema multiforme. The response to salicylates was striking.

Observation III. In January, 1934, a temperature of $103^{\circ} \mathrm{F}$. (rectal) was induced artificially for three hours by means of exposure in a hyperthermia box. This treatment was not followed by any evidence, during subsequent observation, of a clinical recurrence of rheumatic fever. Hemolytic streptococci were absent throughout this observation.

Observation IV. In February, 1934, and seven weeks after the third observation, the patient received for the second time a single injection of $0.1 \mathrm{cc}$. of vaccine which precipitated a slight chill and the usual febrile reaction 
JULY, 1933 NAME R.MeG. FEMALE AGE 16 No.5614

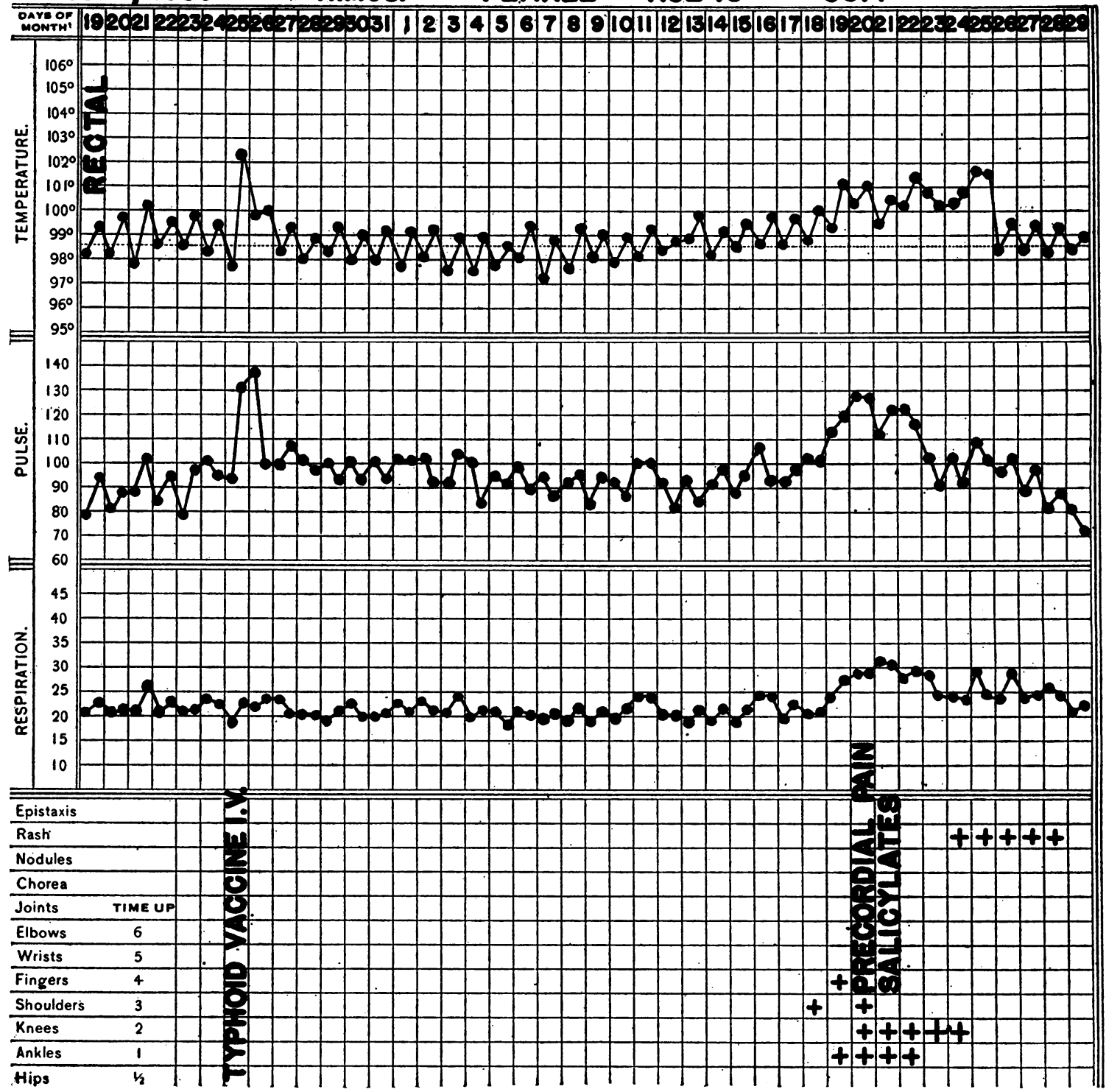

Fig. 5. A Recrudescence of Rheumatic Fever Following Protein Shock in the Same Patient as in FigURE 4.

(Figure 6). In contrast to the previous postvaccinal reaction, an immediate reactivation of the latent rheumatic infection became manifest with the appearance of fever, multiple arthritis, erythema multiforme, and an elevation of the corrected sedimentation index (method of Rourke and Ernstene (13)) from a previously normal level of $0.28 \mathrm{~mm}$. per minute to $1.10 \mathrm{~mm}$. per minute, together with an increase in auriculoventricular conduction time from 0.16 second to 0.19 second. The symptoms subsided spontaneously without salicylate therapy. Hemolytic streptococci were absent throughout this observation. Of considerable interest is the fact that our clinical impression suggests that in spite of the apparently in- duced recrudescence of rheumatic fever this patient's low-grade infection of several years standing was definitely improved following the last episode, so that in May, 1934, all laboratory and clinical evidence of infection had disappeared and she was discharged from the hospital. Her cardiac status remained essentially as at entry.

\section{Case II}

I. P., H.G.S. 5703, female, age 17 , gave a history of rheumatic fever at the age of twelve years, with probable low-grade activity during subsequent winters as indicated by frequent joint pains, poor health and nose- 
fEB.1934 Name R.MeQ. female ACE 17 No. 6814

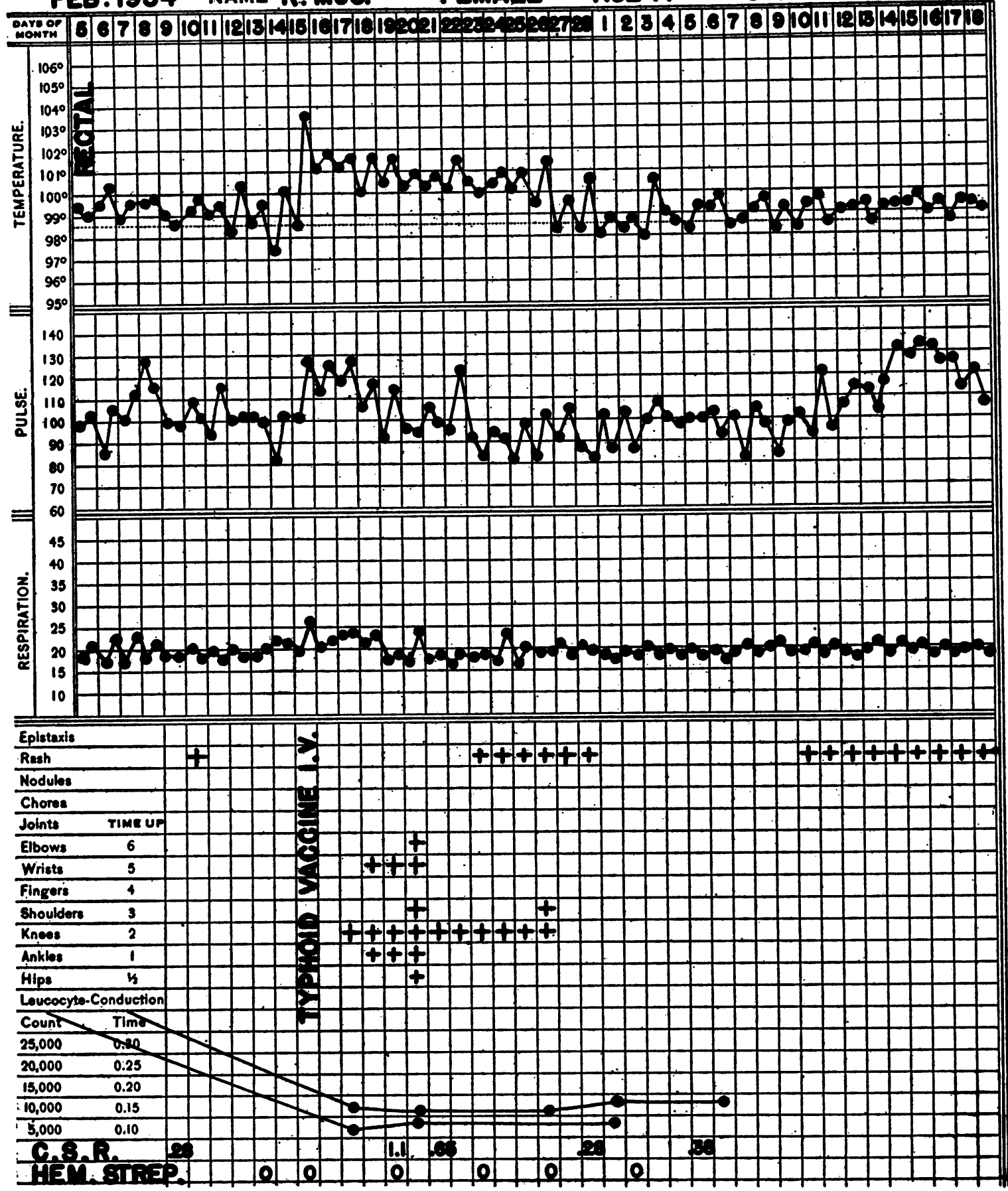

Fig. 6. A Second and Immediate Recrudescence of Rheumatic Fever Following Protein Shock in the Same Patient as in Figures 4 and 5. 


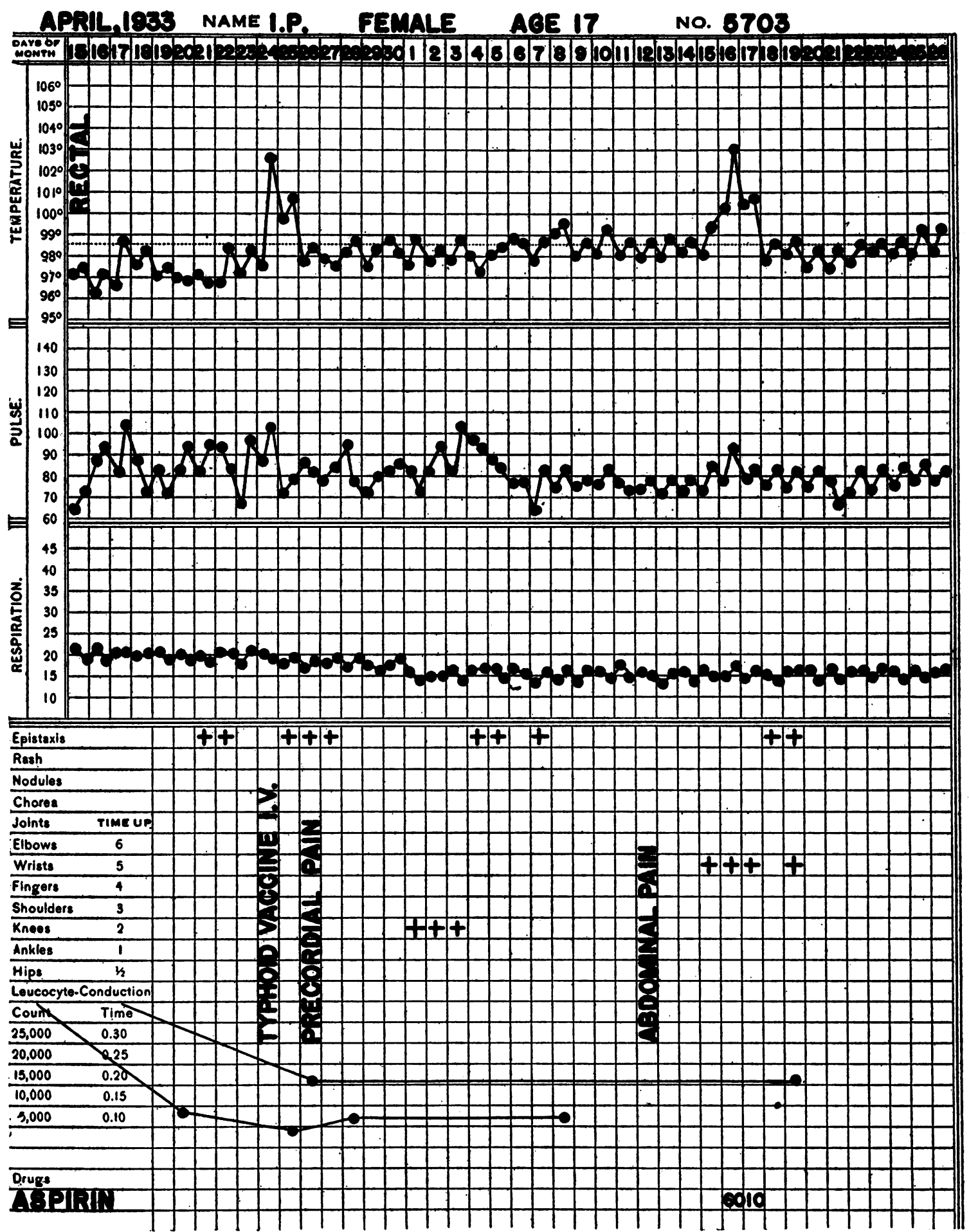

Fig. 7. A Recrudescence of Rheumatic Fever Three Weeks After a Single Protein Shock. 
bleeds. In October, 1932, following a tonsillectomy, she had mild rheumatic fever with symptoms for three weeks. In February, 1933, she had a sore throat and the "grippe." Three weeks later she entered the hospital because of general poor condition and was found to have evidence of active rheumatic infection with fever, precordial pain, occasional nosebleeds, and a well marked elevation of the corrected sedimentation index of 1.40 $\mathrm{mm}$. per minute. The heart was moderately enlarged, with the murmurs of mitral regurgitation and stenosis and aortic regurgitation. She improved with rest in bed, and after one week the temperature returned to normal.

Observation I. In April, 1933, she was given a single intravenous injection of $0.1 \mathrm{cc}$. typhoid-paratyphoid vaccine, which was followed by a transient elevation of temperature and a slight chill (Figure 7). During the subsequent three weeks there occurred frequent slight nosebleeds (present before), vague ankle pains, occasional precordial and abdominal pain. At the end of the third week there appeared a frank flare-up with fever and an acutely swollen, red and tender wrist, which became so painful that salicylates were required. Prompt relief resulted, but a vague discomfort in the wrist and fingers persisted for several days. Throat cultures were not done at this time. The patient was discharged from the hospital in November, 1933, without further occurrence of acute rheumatic fever. The heart remained as at entry.

\section{Case III}

E. F., H.G.S. 5876 , female, age 16 , gave a history of the onset of rheumatic fever and rheumatic heart disease at the age of six years. At the age of thirteen she had a recurrence of rheumatic fever. In January, 1933, at the age of 16 , there was a third attack one week after a sore throat, and she entered the hospital for prolonged bed care. She appeared to be in good general condition, and the only evidence of low-grade infection was an occasional nosebleed and almost daily erythema multiforme. The auriculoventricular conduction time by electrocardiogram remained at $0.20-0.21$ second.

Observation I. In April, 1933, a temperature and associated chill was induced by the intravenous administration of $0.1 \mathrm{cc}$. of typhoid-paratyphoid vaccine as before. A slight rise in temperature to 100 to $100.5^{\circ} \mathrm{F}$. (rectal) persisted for five days, but there was no definite evidence of a subsequent flare-up of the rheumatic process. Hemolytic streptococci were present in throat cultures taken on the day of the protein shock, absent on the fifth day, and again present on the ninth day after the injection. The white blood count and the auriculoventricular conduction time remained unchanged.

Observation II. In January, 1934, an artificial fever of $102.5^{\circ} \mathrm{F}$. (rectal) induced by means of a hyperthermia box was not followed by any clinical manifestations of rheumatic fever. Throat cultures showed no hemolytic streptococci before the procedure. Hemolytic streptococci were present in the throat on the first day after the treatment, and absent on subsequent days.
Observation III. In February, 1934, a single protein shock was induced in the usual manner by the intravenous injection of $0.1 \mathrm{cc}$. of vaccine (Figure 8). This was followed by a low-grade fever for five days associated with a nosebleed and the reappearance of erythema multiforme on the seventh day, but of most interest, perhaps, was the electrocardiographic evidence of slightly delayed but progressively increasing block in auriculoventricular conduction from a $P-R$ interval of 0.18 second up to 0.25 second on the tenth day. From a normal level of $0.25 \mathrm{~mm}$. per minute the corrected sedimentation index rose to $1.60 \mathrm{~mm}$. per minute. Hemolytic streptococci were present in the throat cultures before the protein shock, but absent afterwards. We have considered this a definite recrudescence of rheumatic fever following protein shock.

\section{Case IV}

A. C., H.G.S. 5351, male, age 10 , gave a history of four previous attacks of rheumatic fever and one of chorea during the preceding five years, in spite of which he had developed only minimal rheumatic heart disease, with the murmurs of slight mitral disease together with slight cardiac enlargement. In March, 1933, he again developed rheumatic fever, precipitated by a cold, with polyarthritis, nosebleeds, loss of weight and mild choreiform movements. On April 13, 1933, he entered the hospital for his second admission, where his subsiding joint pains as well as his fever responded promptly to salicylates.

Observation I. As shown in Figure 9, protein shock was induced by the intravenous injection of $0.1 \mathrm{cc}$. of typhoid-paratyphoid vaccine, which gave rise to a severe chill and a rather unusual temperature reaction to $105^{\circ} \mathrm{F}$. (rectal). Within an hour from the onset of the chill two joints of the left hand, which had been involved on admission, became acutely tender, definitely red, hot and swollen. On the second day another finger and the wrist joint showed similar involvement. Because of the persistent and brisk temperature reaction, together with the considerable joint discomfort, salicylates were started with a prompt and striking subsidence of both fever and arthritis, even with minimal doses. Slight joint pains and an occasional slight fever persisted during the subsequent two weeks. It is of interest that the mild choreiform movements became quiescent after the febrile reaction. The corrected sedimentation index on April 28 was $1.40 \mathrm{~mm}$. per minute. A study of the pharyngeal flora was not made during this episode. There were no further recrudescences, and by June, 1933, all clinical and laboratory evidence of infection had subsided. The heart remained as at entry. It is to be noted that clinical evidence of rheumatic fever was present in this patient at the time of the protein shock, and was manifested by recently swollen and painful joints which had been relieved by salicylates, by mildly active chorea and by a leukocytosis of 16,000 . 


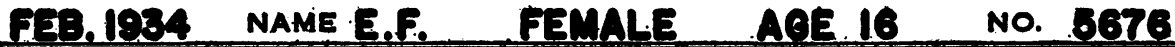

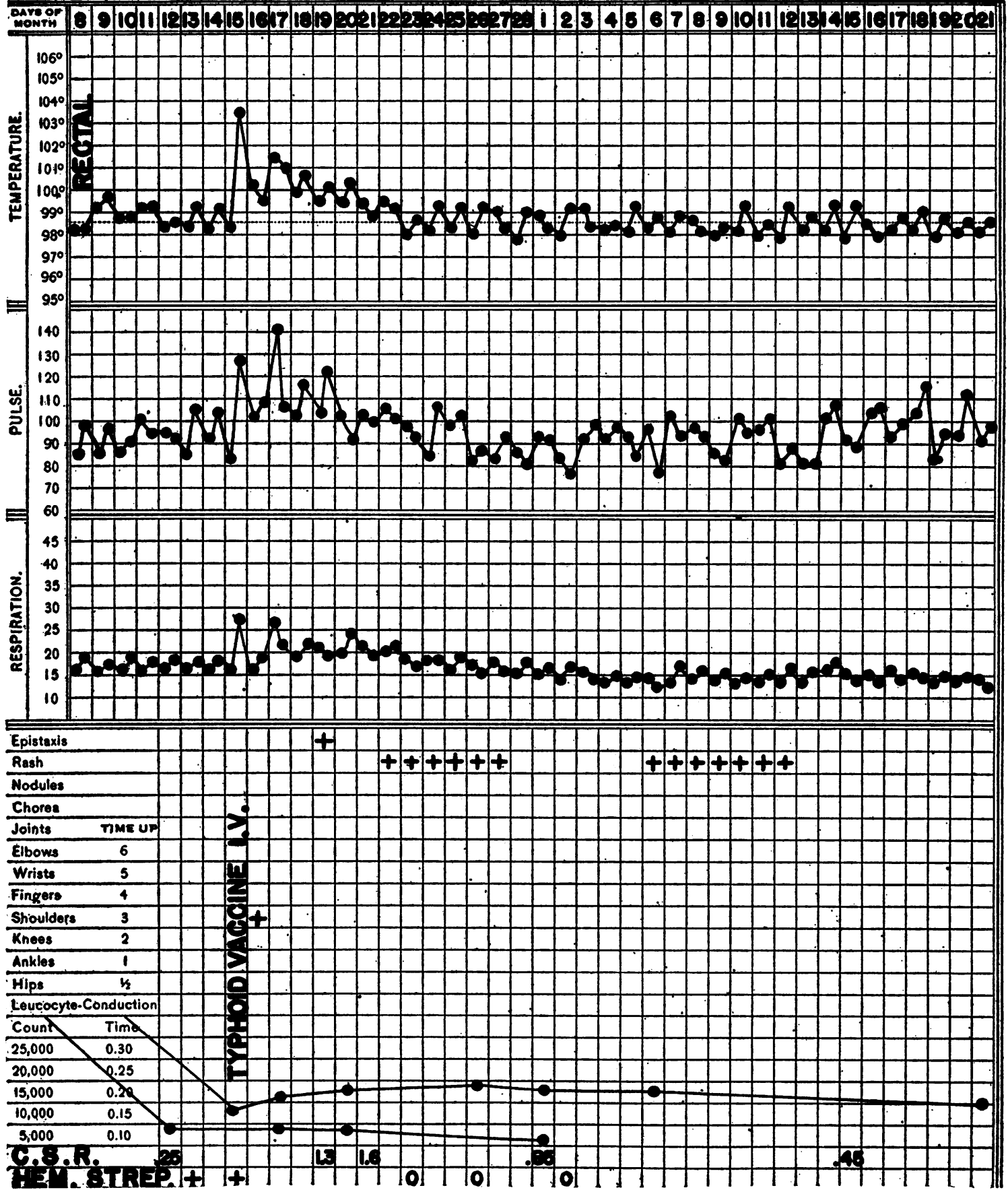

Fig. 8. An Apparent Reactivation of Rheumatic Fever Following Protein Shock.

Of unusual interest here is the slightly delayed but progressive increase in the auriculoventricular conduction time by electrocardiogram from 0.18 second to 0.25 second. 


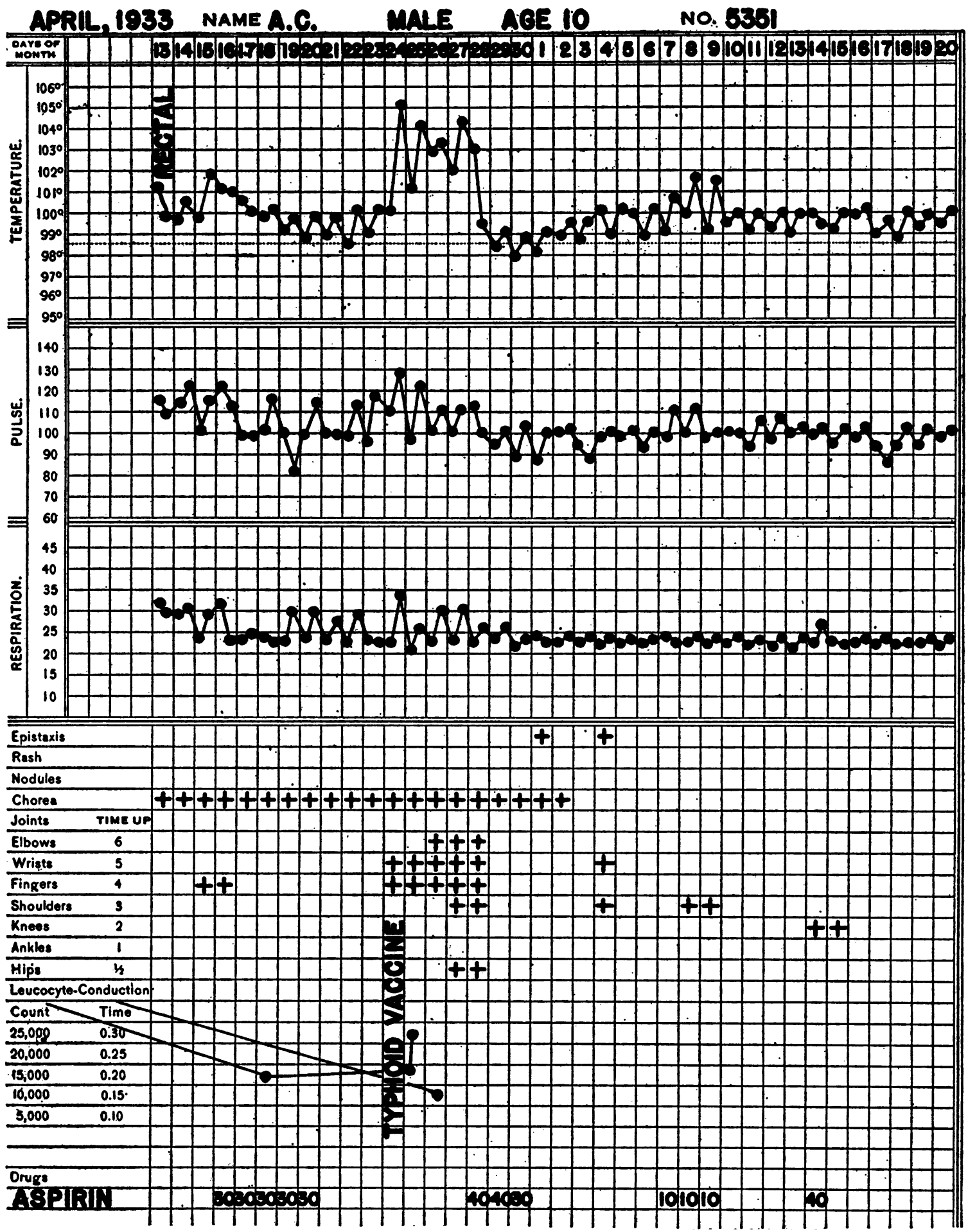

Fig. 9. An Immediate Recrudescence of Rheumatic Fever Following Protein Shock. 


\section{FURTHER OBSERVATIONS}

In addition to, and concurrently with, the above recorded observations, five other patients of similar ages with either low-grade subclinical rheumatic fever or recently quiescent infection received a single protein shock by means of an intravenous injection of $0.1 \mathrm{cc}$. of typhoid-paratyphoid vaccine. The general reaction with chill and fever was similar in all respects, but subsequent clinical and laboratory study failed to reveal frank evidence of a reactivation of the rheumatic process. In two instances there was a short period in which doubtful signs and symptoms suggestive of rheumatic fever occurred. In one of the above instances a comparable fever produced by means of the hyperthermia box was not followed by any evidence of a recrudescence.

In summary, it may be stated that ten patients have received twelve injections of typhoid-paratyphoid vaccine. The usual chill and temperature were noted. In six instances clinical symptoms of active rheumatic fever appeared either immediately following the injection or after an interval of two to three weeks. In two instances there was a questionable reaction of this type. In the remaining four instances no clinical or laboratory evidence of a reactivation of rheumatic fever was demonstrable. No untoward effects of serious import were noted. Following the reactions to typhoid-paratyphoid vaccine there seemed to be a more rapid progress to quiescent rheumatic fever than was previously noted.

\section{DISCUSSION}

Respiratory infection and its relation to rheumatic fever has for years received much attention. The recurrence of rheumatic fever following respiratory infection, often after an interval of a few days to two or three weeks, is generally known to students of the disease. It is also well recognized that recurrences are observed in the absence of evident previous infection or of other disturbing factors. In our series there has been a preceding respiratory infection in 75 per cent of observed recurrences; whereas, in the remaining 25 per cent there were no obvious precipitating causes. On the other hand it may be well to point out that attacks of definite tonsillitis or pharyngitis are not consistently followed by re- currences of rheumatic fever. That other events, such as tonsillectomy, may at times be associated with subsequent rheumatic fever has also been noted in the past. Attention is called to a variety of episodes which appear to have been related to recurrent rheumatic fever. If one omits scarlet fever and other infections usually associated with infection of the upper respiratory tract, there still remain other events which appear to be of importance. Under these circumstances, a reliable history must be obtained soon after the event has transpired, as is often necessary even in the case of respiratory infections, since transient minor illness or injury may be quickly forgotten by the patient. Although it is obvious that the relative number of such events is small in so large a group of cases, no statistical study has been presented for two reasons: first, a history from this point of view is reliable only if one is cognizant of the possibility of such a relationship; and second, as noted above, it is necessary to obtain the history while events are fresh in the patient's mind. The majority of our patients have had rheumatic fever for a considerable period of time prior to admission to the hospital, and only recently has this sequence of events been searched for. Second, in a hospital such as ours traumatic accidents are not observed, nor are illnesses other than rheumatic fever common. A reliable statistical study, although difficult to obtain, we believe would be valuable. Since we have been aware of the possible importance of events other than streptococcal infections prior to recurrences of rheumatic fever, the frequency with which they have been encountered has increased surprisingly.

It is not rare to find instances in which an abdominal operation is a precursor of clinical rheumatic fever. This would seem to hold, regardless of the need for the surgical procedure or the diagnosis for which it is undertaken.

It seems evident to us that it is not rare for events other than respiratory infection to precede the appearance of clinical rheumatic fever, and that they must be considered in some manner a precipitating factor, possibly non-specific.

The clinical observation of the reappearance of the symptoms of rheumatic fever following a single treatment with non-specific protein therapy 
seems of considerable importance to us. Rheumatic fever was not produced in normal human beings, but the asymptomatic phase of the disease was brought to a clinical level in known rheumatic individuals by non-specific means. The frequency and definiteness of these few observations convince us that we are dealing with more than a chance relationship. The studies have not been pursued further for the obvious reason that we did not wish to cause clinical disturbances in our patients. It is generally accepted that subjects with rheumatic fever, especially those with recent histories of rheumatic fever, are very susceptible to recurrences. It is evident that these may be precipitated frequently by purely non-specific means. These observed recurrences were, on the whole, of shorter duration and were milder than those occurring naturally. No evident alteration of the cardiac state, other than the temporary prolongation of the auriculoventricular conduction time, was noted. The symptoms noted during the recurrences of clinically active rheumatic fever, following the reaction to a single injection of nonspecific protein, reproduced in a striking fashion the rheumatic manifestations which had been previously observed in the individual patient during a naturally occurring recrudescence.

As noted previously, the initiating factor most commonly encountered in patients with rheumatic fever is respiratory infection. In view of the data presented, bacterial activity during respiratory infection may prove to be of secondary importance. The crowded, unhygienic living conditions of the majority of patients with rheumatic fever may well account for the frequency of respiratory infections. In addition, the study of any group of patients with rheumatic fever will show a large number of such respiratory infections even under satisfactory living conditions.

In view of the observations herein recorded, it is suggested that the rôle of infections or of episodes seemingly related to recurrent rheumatic fever may not be as specific as has been previously thought. Respiratory infection, often accompanied by hemolytic streptococci, is frequently the precipitating agent of rheumatic fever, but other events likewise may be important. The appearance of the clinical symptoms and signs of the disease are not, therefore, entirely dependent upon recognizable respiratory infection. This apparently non-specific relationship may be of importance in future etiological considerations.

\section{CONCLUSIONS}

1. There appears to be no significant clinical difference between the recurrences or recrudescences of rheumatic fever following (1) respiratory infection, (2) other forms of infection, (3) accidents or operative procedures, and (4) a single intravenous injection of typhoidparatyphoid vaccine sufficient to cause a slight febrile reaction and chill.

2. The probable significance of these observations has been discussed. It is evident that various events precede and apparently influence the appearance of the signs and symptoms of recurrent rheumatic fever.

3. It seems desirable, in view of the observations presented, to consider the rôle of such events as non-specific until more definite information is available concerning the etiological agent.

We wish to gratefully acknowledge our indebtedness to Doctors James H. Means and M. N. Smith-Petersen of the Massachusetts General Hospital for allowing us to cite cases from their respective services. The typhoid-paratyphoid vaccine used throughout these observations was obtained from the Antitoxin Laboratory of the Massachusetts Department of Public Health.

\section{BIBLIOGRAPHY}

1. Coburn, A. F., The Factor of Infection in the Rheumatic State. Williams \& Wilkins, Baltimore, 1931.

2. Coburn, A. F., and Pauli, R. H., Studies on the relationship of streptococcus hemolyticus to the rheumatic process. I. Observations on the ecology of hemolytic streptococcus in relation to the epidemiology of rheumatic fever. J. Exper. Med., 1932, 56, 609.

3. Schlesinger, B., The relationship of throat infection to acute rheumatism in childhood. Arch. Dis. Childhood, 1930, 5, 411.

4. Collis, W. R. F., Acute rheumatism and haemolytic streptococci. Lancet, 1931, 1, 1341.

5. Sheldon, W., On acute rheumatism following tonsillitis. Lancet, 1931, 1, 1337.

6. Gibson, H. J., Thomson, W. A. R., and Stewart, D., The haemolytic streptococcus as a factor in the 
causation of acute rheumatism. Arch. Dis. Childhood, 1933, 8, 57.

7. Boas, E. P., and Schwartz, S. P., Some modes of infection in rheumatic fever. Am. Heart J., 1927, 2, 375.

8. Hiller, R. I., and Graef, I., An epidemic of rheumatism at a cardiac camp. Am. Heart J., 1928, 3, 271.

9. Zinsser, Hans, On the significance of bacterial allergy in infectious diseases. Bull. N. Y. Acad. Med., $1928,4,351$.
10. Swift, H. F., Rheumatic fever. J. A. M. A., 1929, 92, 2071.

11. Sutton, L. P., and Dodge, K. G., The treatment of chorea by induced fever. J. Ped., 1933, 3, 813.

12. Sutton, L. P., and Dodge, K. G., The effect of fever therapy on rheumatic carditis associated with chorea. J. Pediat., 1935, 6, 494.

13. Rourke, M. D., and Ernstene, A. C., A method for correcting the erythrocyte sedimentation rate for variations in the cell volume percentage of blood. J. Clin. Invest., 1930, 8, 545. 